\title{
An Integrated Mosquito Surveillance Module in New York State
}

\author{
Hwa-Gan Chang ${ }^{\star 1}$, Jacqueline Griffin ${ }^{2,1}$, Charlie DiDonato ${ }^{2,1}$, Cori Tice ${ }^{1}$ and \\ Bryon Backenson'
}

${ }^{1} \mathrm{NYSDOH}$, Albany, NY, USA; ${ }^{2 N T T}$ Data, Albany, NY, USA

\section{Objective}

To develop a mosquito surveillance module to collect mosquito information testing for West Nile, East Equine Encephalitis (EEE) and Zika viruses using national standards. To provide a common set of data for local health departments (LHDs) and state users to report and share information. To monitor the type of mosquito species that carry diseases.

\section{Introduction}

There were several stand-alone vector surveillance applications being used by the New York State Department of Health (NYSDOH) to support the reporting of mosquito, bird, and mammal surveillance and infection information implemented in early 2000s in response to West Nile virus. In subsequent years, the Electronic Clinical Laboratory Reporting System (ECLRS) and the Communicable Disease Electronic Surveillance System (CDESS) were developed and integrated to be used for surveillance and investigations of human infectious diseases and management of outbreaks.

An integrated vector surveillance system project was proposed to address the migration of the stand-alone vector surveillance applications into a streamlined, consolidated solution to support operational, management, and technical needs by using the national standards with the existing resources and technical environment.

\section{Methods}

A mosquito surveillance module was designed to link with CDESS, an electronic disease case reporting and investigation system, to allow LHDs to enter mosquito trap sites and mosquito pool information obtained from those traps. The mosquito test results are automatically transmitted to ECLRS through public health lab Clinical Laboratory Information Management System (CLIMS) using ELR standards. By utilizing these standards, the ECLRS was enhanced to add a new nonhuman specimen table and existing processes were used to obtain mosquito laboratory results and automatically transfer them to the surveillance system the same way that human results are transferred. The new mosquito surveillance module also utilizes the existing CDESS reporting module, thereby allowing users the flexibility to query and extract data of their choosing. The minimum infection rate (MIR) report calculates the number of infected pools with an arbovirus divided by the total number of specimens tested*1000; a trap report shows number of mosquitoes trapped by species type, location and trap type; and a lab test result report shows the number of pools that tested positive and the percentage of positive pools by disease.

\section{Results}

The mosquito surveillance module was rolled out in May 2016 to all 57 LHDs. A non-human species lookup table was created to allow public health lab to report the test results using Health Level seven (HL7) v 2.5.1 standards. As of August 31, 2016 there were 4,545 pools tested. A total of 201 (4.4\%) pools were positive for West Nile and the MIR was 1.2. There were no pools positive for EEE or Zika virus. Various reports have been created for monitoring the surveillance of mosquitoes trapped and tested for mosquito-borne diseases.

\section{Conclusions}

The integration of mosquito surveillance module within CDESS allows LHDs and the State to monitor mosquito-borne disease activity more efficiently. The module also increases NYDOH's ability to provide timely, accurate and consistent information to the local health departments and healthcare practitioners regarding mosquito-borne diseases.

\section{Keywords}

information system; mosquito surveillance; national standards

\section{*Hwa-Gan Chang}

E-mail: hwagan.chang@health.ny.gov 